\title{
Extended use of raltegravir in the treatment of HIV-I infection: optimizing therapy
}

This article was published in the following Dove Press journal:

Infection and Drug Resistance

12 October 2010

Number of times this article has been viewed

\author{
Charlotte Charpentier' \\ Laurence Weiss ${ }^{2}$ \\ 'Assistance Publique-Hôpitaux \\ de Paris, Hôpital Bichat-Claude \\ Bernard, Laboratoire de Virologie, \\ Université Paris-Diderot, Paris, \\ France; ${ }^{2}$ Assistance Publique-Hôpitaux \\ de Paris, Hôpital Européen Georges \\ Pompidou, Service d'Immunologie \\ Clinique, Université Paris Descartes, \\ Paris, France
}

\begin{abstract}
Raltegravir is the first licensed compound in 2007 of the new integrase inhibitor drug class. At the dose of $400 \mathrm{mg}$ twice daily, raltegravir showed a potent antiviral action in antiretroviral-naïve patients when associated with tenofovir and emtricitabine. Raltegravir was also found to be highly active in antiretroviral-experienced patients with virological failure and displaying multiresistant virus, as shown with the BENCHMRK and ANRS 139 TRIO trials. Finally, the use of raltegravir was assessed in the context of a switch strategy in antiretroviralexperienced patients with virological success [human immunodeficiency virus type 1 (HIV-1) RNA below detection limit], highlighting the following mandatory criteria in this strategy: the nucleoside reverse transcriptase inhibitors associated with raltegravir have to be fully active. In the different studies, raltegravir had a favorable safety and tolerability profile. In the clinical situation a switch in virologically suppressed patients receiving a protease inhibitor, an improvement of the lipid profile was observed. Overall, when analyzing the Phase II and III trials together, only a few patients on raltegravir discontinued for adverse events. The development of resistance to raltegravir mainly involved three resistance mutations in integrase gene: Q148H/K/R, N155H, and $\mathrm{Y} 143 \mathrm{C} / \mathrm{H} / \mathrm{R}$. In conclusion, raltegravir improved the clinical management of HIV-1 infection both in antiretroviral-naïve and in antiretroviral-experienced patients.
\end{abstract}

Keywords: HIV-1, integrase inhibitors, raltegravir, antiretroviral therapy

\section{Rationale for the development of new antiretroviral drugs in HIV infection management}

Since 1996, highly active antiretroviral therapy is still the standard of care for patients infected with human immunodeficiency virus (HIV) and displaying advanced immunodeficiency. ${ }^{1}$ Combination regimens have resulted in improved survival and decreased morbidity for patients with a CD4 cell count below $350 \mathrm{cells} / \mathrm{mm}^{3}{ }^{2}$ However, viral suppression cannot always be achieved or sustained with standard antiretroviral-based regimens because of the development of viral resistance, toxic effects of drugs, drug intolerance, or problems of adherence. ${ }^{3}$ The majority of HIV-infected patients in whom highly active antiretroviral therapy fails displayed plasma-resistant virus. ${ }^{4}$ When only two viral enzymes (ie, reverse transcriptase and protease) were targeted by antiretroviral drugs, cross-resistance rapidly limits therapeutic options. Thus, antiretroviral drugs directed at new HIV targets were urgently needed for patients exhibiting detectable viremia despite treatment.

In this context, HIV type 1 (HIV-1) integrase represented one of the possible new therapeutic target. ${ }^{5,6}$ Consequently, HIV-1 integrase inhibitors would be expected to retain activity against HIV-1 that is resistant to other classes of antiretroviral 
drugs. In October 2007, raltegravir (MK-0518; Isentress, Merck, Whitehouse Station, NJ) was the first approved HIV-1 integrase inhibitor; it targets the strand transfer step of HIV-1 integration. It is currently approved by the US Food and Drug Administration in a large indication: "in combination with other antiretroviral agents for the treatment of HIV-1 infection in adult patients" without any specific criteria, both in antiretroviral-naïve and in antiretroviralexperienced patients.

Twenty years after the discovery of the therapeutic activity of zidovudine, the first antiretroviral drug used in the clinic, more than 25 antiretroviral drugs are now available, representing six different drug classes.

\section{Overview of pharmacology and mechanism of action of raltegravir Mechanism of action}

The complete viral life cycle requires integration of HIV-1 viral DNA into the host cell genome. It occurs in three steps (Figure 1). ${ }^{5,7,8}$ The double-stranded DNA copy generated by reverse transcription of the HIV-1 viral RNA genome is a part of the "preintegration complex", containing both cellular and viral proteins, including integrase. In a first step, termed ' 3 ' processing", two nucleotides are removed from the 3 ' ends of the viral DNA. In a second step, termed "strand transfer", the proviral DNA is inserted into the host DNA and joined with it. Then, gaps in DNA are repaired by cellular enzymes by removing the two unpaired nucleotides at the $5^{\prime}$ end of the proviral DNA. The HIV integrase catalyses all steps of integration process except the last one, ie, DNA repair and ligation. A large series of selective inhibitors of strand transfer, $\beta$-diketo acid derivates, were tried to be developed as orally bioavailable agents. ${ }^{6,9-12}$ Mechanistic studies showed that L-731,988, a $\beta$-diketo acid derivate, recognizes and selectively binds the integrase donor substrate complex exclusively in the context of catalytically active protein assembled on the viral DNA end. Thus, L-731,988 binds within the integraseactive site and inhibits strand transfer by competing with the target DNA substrate. ${ }^{13}$

Chemical optimization of these compounds led to naphthyridine derivatives and L-900612 or MK-0518 (raltegravir) as a promising candidate compound. A recent study based on the diffracting crystals of the full-length integrase from the prototype foamy virus in complex with its cognate viral DNA enabled to determine more precisely the contacts between raltegravir and integrase structure. ${ }^{14}$ The latter particularly included the metal cofactors of the active site, the bases of the invariant $\mathrm{CA}$ dinucleotide, and integrase residues 212,214 , and 215 . Thus, the mechanism of action of the integrase strand-transfer inhibitors was more precisely described. Thus, the drug binding to integrase leads to the displacement of the reactive viral DNA end from the active site, disarming the viral nucleoprotein complex. ${ }^{14}$ This could explain why the integrase strand-transfer inhibitors preferentially interact with and inhibit the DNA-bound form of HIV-1 integrase. ${ }^{14}$

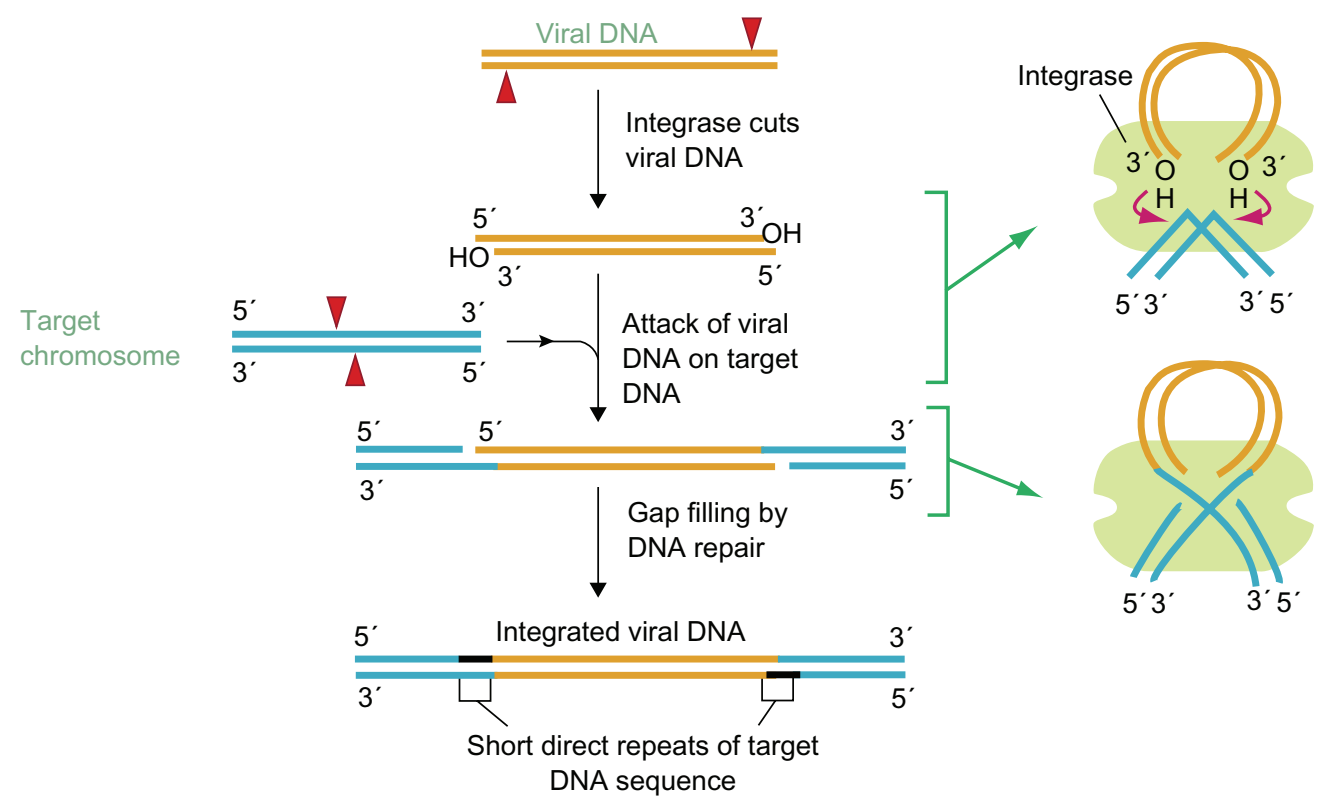

Figure I Steps of viral integration. Copyright (C) 2002. Reproduced with permission from NCBI DNA Replication, Repair, and Recombination. In: "Basic Genetic Mechanisms", Alberts B, Johnson A, Lewis J, Raff M, Roberts K, Walter P, editors. Molecular Biology of the Cell. New York, NY: Garland Science; 2002. 
Raltegravir has potent in vitro activity against HIV-1, with a $95 \%$ inhibitory concentration (IC95; \pm SD) in human $\mathrm{T}$ lymphoid cell cultures of $31 \pm 20 \mathrm{nmol} / \mathrm{L}$. It is active against a wide range of wild-type and drug-resistant HIV-1 isolates harboring resistance mutations in reverse transcriptase and protease coding regions, including both virus using CCR5 coreceptor and CXCR4 coreceptor. ${ }^{9}$

The in vivo activity of raltegravir was first described in the Phase II protocol 004, in which increasing doses of 100 , 200, 400, and $600 \mathrm{mg}$ of raltegravir administrated twice daily were compared with the placebo. ${ }^{15}$ Thirty-five subjects received a 10-day course of raltegravir monotherapy at the doses described earlier or placebo. After 10 days, the mean HIV-1 RNA decrease was 1.66-2.16 $\log _{10}$ copies/mL in raltegravir recipients as compared with $0.17 \log _{10}$ copies $/ \mathrm{mL}$ in placebo-treated patients. No significant difference in HIV-1 RNA decrease was evidenced in the different raltegravir arms (Figure 2).
Thus, in vitro and in vivo activities of raltegravir against HIV-1 were demonstrated. Furthermore, additive or synergistic activity was observed when cell culture samples, infected with HIV-1, were incubated with raltegravir and a panel of available nucleoside analog reverse transcriptase inhibitors (NRTI), nonnucleoside reverse transcriptase inhibitors (NNRTI), and protease inhibitors (PI). Taken together, these findings argue for the use of raltegravir with other active antiretroviral drugs.

\section{Pharmacology of raltegravir}

Oral absorption of raltegravir is rapid with a median time to maximum concentration of $0.5-1.3$ hours, and oral bioavailability is approximately $30 \% .{ }^{16}$ Plasma protein binding reaches $83 \%$. There was a biphasic decrease in drug concentrations, with an initial-phase half-life of $\sim 1$ hour and a terminal-phase half-life of 7-12 hours (Merck). These characteristics were not affected by food
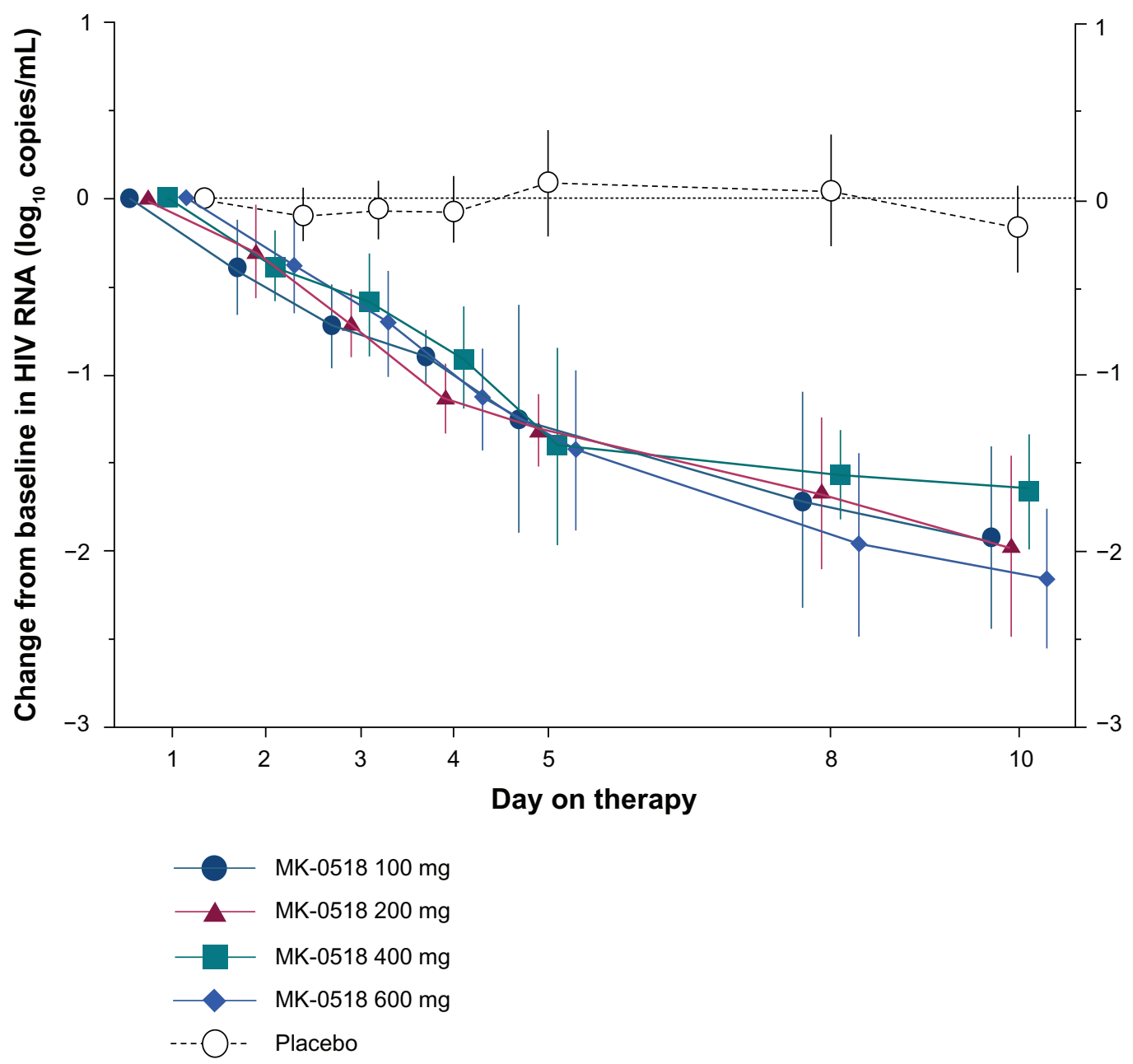

Figure 2 Change from baseline at day 10 in HIV-I RNA $\log _{10}$ copies $/ \mathrm{mL}$ (with $95 \% \mathrm{Cl}$ ). Error bars indicate $95 \% \mathrm{Cl}$. Data for a total of 7 patients (from 4 of the 5 treatment groups) were not available on day 5. Copyright @ 2007. Adapted with permission from Markowitz M, Nguyen BY, Gotuzzo E, et al. Rapid and durable antiretroviral effect of the HIV-I integrase inhibitor raltegravir as part of combination therapy in treatment-naïve patients with HIV-I infection: results of a 48-week controlled study. J Acquir Immune Defic Syndr. 2007;46(2): I25-133. 
with moderate-to-high fat content. Raltegravir is mainly metabolized by the glucuronidation enzyme UGT1A1, not by the CYP450 enzyme system. Thus, the potential for drug-drug interactions is decreased and administration of raltegravir may be of interest in combination with antituberculosis drugs or immunosuppressive agents, for example, with the exception of rifampicin. Thus, rifampicin is the inducer of UGT1A1, resulting in lower plasma raltegravir concentrations and needing to double the raltegravir dose to $800 \mathrm{mg} .{ }^{17}$ Finally, excretion in feces and in urine accounts for most of the elimination.

Raltegravir is administered twice daily. No dose adjustments are recommended for severe renal impairment or mild-to-moderate hepatic impairment. The independence of the compound from "boosting" of drug levels with ritonavir is an attractive feature for many patients suffering from ritonavir-associated side effects. Interestingly, atazanavir inhibits UGT1A1, thus atazanavir is a likely combination partner for raltegravir, and pharmacokinetic studies with atazanavir, with or without ritonavir, showed a 30\%-70\% increase in raltegravir area under the plasma concentration time curve (AUC). ${ }^{18}$ However, further studies are needed to assess the clinical relevance of this mechanism.

\section{Efficacy studies}

Raltegravir-containing regimens were demonstrated to have potent antiretroviral activity and to be well tolerated in HIV-1-infected individuals. Clinical trials were performed in different groups of HIV-1-infected patients, ie, treatmentnaïve patients and antiretroviral-experienced patients with virological failure and exhibiting multiresistant plasma virus. Finally, the use of raltegravir was also assessed in switch strategy in antiretroviral-experienced patients with virological success.

\section{Treatment-naïve patients}

Patients included in the 10-day course of raltegravir monotherapy protocol (protocol 004) described earlier $(\mathrm{n}=30)$ and 171 additional patients were randomized to receive either raltegravir $(100,200,400$, or $600 \mathrm{mg}$ twice daily) or efavirenz in combination with tenofovir and lamivudine for 48 weeks. ${ }^{15}$ At baseline, mean viral load was 4.7 and $4.8 \log _{10}$ copies/mL and mean CD4 cell count was 280 and 305 cells $/ \mathrm{mm}^{3}$ in the raltegravir and efavirenz arms, respectively. At week 24, the HIV-1 RNA level was $<50$ copies $/ \mathrm{mL}$ in $85 \%-98 \%$ of raltegravir recipients and in $92 \%$ of efavirenz recipients. The mean change in CD4 cell count ranged from 144 to 221 cells $/ \mathrm{mm}^{3}$ across all raltegravir groups at week 48. After week 48, all patients in the raltegravir arms were given $400 \mathrm{mg}$ twice daily. The virological response was sustained up to 96 weeks. ${ }^{19}$

This latter study confirmed the efficacy of raltegravir at the dose of $400 \mathrm{mg}$ twice daily in antiretroviral-naïve patients, allowing to initiate Phase III trials using raltegravir-based regimen in first-line.

The STARTMRK study was an international, doubleblind, Phase III randomized, noninferiority trial. ${ }^{20}$ The study compared the safety and efficacy of raltegravir vs efavirenz in combination with tenofovir and emtricitabine in treatment-naïve patients. Inclusion criteria are as follows: HIV-1 infected patients naïve of antiretroviral treatment with HIV-1 RNA > 5000 copies/mL. Most patients were at an advanced stage of disease. Thus, 267 (47\%) patients had a CD4 cell count of $\leq 200$ cells $/ \mathrm{mm}^{3}$ at baseline, of whom 58 $(22 \%)$ had $\leq 50$ cells $/ \mathrm{mm}^{3}$. More than half $[\mathrm{n}=297(53 \%)]$ of participants had HIV-1 RNA level of $>5 \log _{10}$ copies/mL.

In the main analysis that recorded as failures all patients who did not complete the study, 86.1\% $(n=241)$ of the raltegravir group achieved the primary endpoint of $<50$ copies/mL at week 48 , compared with $81.9 \%(n=230)$ of the efavirenz group [difference $4.2 \%, 95 \%$ confidence interval (CI): -1.9 to 10.3 ], indicating that raltegravir was noninferior to efavirenz ( $P<0.0001$ for noninferiority). The mean change in CD 4 cell count at week 48 was 189 cells $/ \mathrm{mm}^{3}$. The noninferiority of the raltegravir arm was also demonstrated in patients exhibiting HIV-1 RNA $>5 \log _{10}$ copies $/ \mathrm{mL}$. Interestingly, the time to achieve such viral suppression was shorter for patients on raltegravir than those on efavirenz (log-rank test $P<0.0001$ ) (Figure 3); however, the clinical significance of a more rapid HIV-1 RNA decline has not yet been established but might be of interest to limit the selection of drug-resistant variants during the phase of viral load decay. $^{21}$

The results of the STARTMRK study at week 96 have recently been published and showed that the non-inferiority of raltegravir relative to efavirenz was sustained (difference $2 \%, 95 \% \mathrm{CI}:-4$ to 9$).{ }^{22}$ Thus, raltegravir combined with tenofovir and emtricitabine is a durably efficacious regimen for treatment-naïve patients.

The results of the STARTMRK study led to the approval of raltegravir in antiretroviral-naïve patients.

\section{Treatment-experienced patients with virological failure}

Protocol 005 was a multicenter, randomized, double-blind, placebo-controlled dose-ranging trial in treatment-experienced 


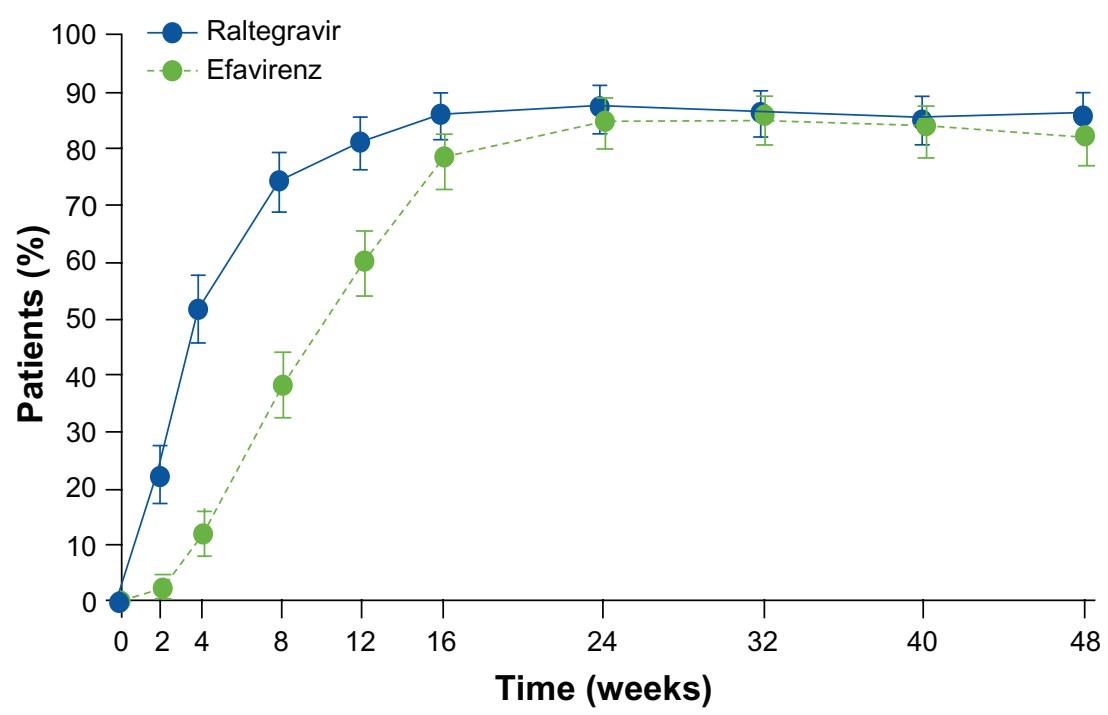

$\begin{array}{rlllllllll}\text { Numbers contributing data } & & & & & & & & & \\ \text { Raltegravir group } & 281 & 279 & 281 & 279 & 281 & 279 & 278 & 280 & 280 \\ \text { Efavirenz group } & 282 & 282 & 282 & 282 & 281 & 282 & 280 & 281 & 281\end{array}$

Figure 3 Proportion of patients with HIV-I RNA $<50$ copies $/ \mathrm{mL}$. Patients who did not complete the study were recorded as failures. Error bars $=95 \% \mathrm{Cl}$. Copyright (C) 2009. Adapted with permission from Lennox JL, Dejesus E, Lazzarin A, et al. Safety and efficacy of raltegravir-based versus efavirenz-based combination therapy in treatment-na naïve ve patients with HIV-I infection: a multicentre, double-blind randomised controlled trial. Lancet. 2009;374(9692):796-806.

patients. ${ }^{23}$ Inclusion criteria were as follows: HIV-1 infected patients with HIV-1 RNA > 5000 copies/mL and CD4 $<50$ cells $/ \mathrm{mm}^{3}$, on stable antiretroviral therapy for more than 3 months and infected with HIV-1 with documented genotypic or phenotypic resistance to at least one NNRTI, one NRTI, and one PI. One hundred seventyeight patients were included in this protocol. Investigators selected an optimal background regimen (OBR) according to all resistance tests available during therapeutic history. Randomization was performed in a $1: 1: 1: 1$ fashion to raltegravir doses of 200, 400, and $600 \mathrm{mg}$ twice daily, or placebo, stratified by PI resistance at baseline and prior enfuvirtide use. Primary end-points were response and toxicity on week 24. Virological response was significantly better for all raltegravir arms as compared to placebo, with no significant difference between the raltegravir arms. This difference in favor of raltegravir was observed with any extent of resistance to the compounds of the background regimen, as assessed by genotypic or phenotypic sensitivity scores corresponding to the number of drugs received by the patient predicted to be active at baseline. Use of additional enfuvirtide was also associated with a better virological outcome. The mean change in CD4 cell count from baseline to week 24 was 113 cells $/ \mathrm{mm}^{3}$ (95\% CI: 73-150), with raltegravir $400 \mathrm{mg}$ vs 5 cells $/ \mathrm{mm}^{3}$ in the placebo group. There was no obvious difference of toxicity profile for any raltegravir dose in comparison with placebo.
The results of the protocol 005 confirmed the efficacy of raltegravir at the dose of $400 \mathrm{mg}$ twice daily in antiretroviral-experienced patients, allowing to initiate Phase III clinical trials using raltegravir as part of a salvage regimen in patients with prior virological failures and exhibiting multidrug resistant virus.

Clinical trials BENCHMRK-1 (performed in Europe, Asia, the Pacific, and Peru) and BENCHMRK-2 (performed in the United States) were two parallel, double-blind, placebocontrolled studies. ${ }^{24-26}$ In both trials, an investigator-selected, resistance analysis-based OBR was combined with either raltegravir or placebo. Randomization was performed in a 2:1 manner. Four hundred sixty-two patients displaying tripleclass resistant virus at baseline and exhibiting plasma HIV-1 RNA levels above 1000 copies/mL were included. The primary end-point was viral suppression to HIV-1 RNA $<400$ copies/mL at week 16, with virus suppression to HIV-1 RNA $<50$ copies/mL and change from baseline viral load and CD4 cell count evaluated as secondary end-points. The raltegravir arms in both trials were superior over placebo with regard to all end-points. The difference was maintained irrespective of the extent of baseline resistance (as assessed by genotypic and phenotypic sensitivity scores), baseline HIV-1 RNA levels, and baseline CD4 cell count. A superior response of raltegravir was sustained until week 24 in a combined analysis of both trials and confirmed after 48 weeks of follow-up. The difference in favor of raltegravir persisted regardless of the 
background regimen. The mean change in CD4 cell count between baseline and week 48 was 109 cells $/ \mathrm{mm}^{3}(95 \%$ CI: 98-121) in the raltegravir recipients as compared with 45 cells $/ \mathrm{mm}^{3}$ in the placebo recipients. The response rate (HIV-1 RNA $<50$ copies $/ \mathrm{mL}$ ) in the raltegravir group at week 48 was $89 \%$ in the subgroup of patients receiving raltegravir in association with enfuvirtide and darunavir, when both drugs were used for the first time. ${ }^{24}$ This approaches response rates in previously untreated patients and therefore represents a marked improvement for clinical management of patients with resistant virus. Results from combined BENCHMRK-1 and BENCHMRK-2 studies after 96 weeks of follow-up have been recently published, confirming the long-term efficacy of this strategy. ${ }^{23}$ Thus, $57 \%$ and $61 \%$ of the patients in the raltegravir arm, compared with $26 \%$ and $28 \%$ of the patients in the placebo group, sustained an HIV-1 RNA level $<50$ copies/mL and $<400$ copies/mL at week 96, respectively, ( $P<0.001$ for both comparisons), demonstrating a superior and durable virological efficacy of the raltegravir-based regimen.

Another trial assessing raltegravir in HIV-experienced patients in virological failure was the Agence Nationale de Recherches sur le SIDA (ANRS) 139 TRIO trial. ${ }^{24}$ The objective of the ANRS 139 TRIO trial was to assess the virological efficacy and safety of an antiretroviral regimen containing raltegravir and two new drugs: the second generation NNRTI etravirine and darunavir boosted with ritonavir in HIV-1-infected patients who experienced virological failure of a combination antiretroviral therapy and displaying plasma multidrug-resistant virus. Patients enrolled in this noncomparative, open multicenter trial were naïve to the three investigational drugs and had plasma HIV-1 RNA levels $>1000$ copies/mL, a history of virological failure while receiving NNRTI, baseline plasma virus exhibiting $\geq 3$ major PI-resistance mutations and NRTI resistance-associated mutations, and $\geq 3$ darunavir and NNRTI resistance-associated mutations. The primary end point was the proportion of patients with plasma HIV-1 RNA levels $<50$ copies $/ \mathrm{mL}$ at week 24 . A total of 103 patients were included, and $87 \%$ of them received an OBR that included NRTI (86 patients) or enfuvirtide (12 patients). At week 24 , $90 \%$ of patients (95\% CI: 85-96) had an HIV-1 RNA level of $<50$ copies/mL. At week 48, 86\% (95\% CI: 80-93) had an HIV-1 RNA level of $<50$ copies/mL. The median CD4 cell count increase from baseline to week 48 was 108 cells $/ \mathrm{mm}^{3}$ [interquartile range (IQR): 58-169]. Thus, highly antiretroviral-experienced patients harboring multidrug-resistant virus and who have few remaining treatment options may benefit from an antiretroviral therapy regimen containing three new drugs, raltegravir, etravirine, and darunavir/ritonavir, and may achieve high levels of virological suppression comparable to that of treatment-naïve patients.

\section{Switch to raltegravir in treatment- experienced patients with virological success}

The aim of these studies was to evaluate a switch from enfuvirtide (ANRS 138 EASIER trial) or a boosted PI as lopinavir (SWITCHMRK trial) to raltegravir in virologically suppressed patients.

The SWITCHMRK 1 and 2 studies were identically desig ned, double-blind, randomized, Phase III, active-controlled, noninferiority clinical trials. ${ }^{25}$ Patients virologically suppressed, receiving a lopinavir-based regimen for at least 3 months were included from 81 centers in five continents. The aims of the study were to assess the relative effects of a switch from lopinavir to raltegravir vs continuation of lopinavir on different parameters: i) serum lipid concentrations, ii) viral suppression, and iii) adverse events. In the combined analysis, viral suppression to $<50$ copies/mL was achieved by $293(84.4 \%)$ patients in the raltegravir group compared with $319(90.6 \%)$ patients in the lopinavir group at week 24 , failing to establish non-inferiority of raltegravir to lopinavir. The studies were terminated at week 24 because of lower than expected virological efficacy in the raltegravir group compared with the lopinavir group. Overall, 49 patients met the protocol definition of confirmed virological failure, 32 in the raltegravir arm and 17 in the lopinavir arm. It is important to note that $18(56 \%)$ and $4(23 \%)$ patients had a history of virological failure on previous regimens, with the selection of NRTI resistance mutations, in the raltegravir and lopinavir arms, respectively. These results can be explained by the heterogeneous population enrolled in the SWITCHMRK studies. Of note, the inclusion criteria did not take account of genotypic resistance test criteria, or analysis of the patients' antiretroviral history, including the occurrence of prior virological failure. Thus, it was not possible to ensure that the antiretroviral drugs present in the OBR were fully active. This study emphasizes that raltegravir had to be associated with fully active antiretroviral drugs to exclude a functional monotherapy context, which will strongly favor the development of resistance.

The SPIRAL study is a multicenter, noninferiority study assessing the efficacy of a switch from ritonavir-boosted PI to raltegravir in selected patients. ${ }^{27}$ To be included, the patients had to i) receive a ritonavir-boosted PI added to at least two 
antiretroviral drugs and ii) show plasma HIV-1 RNA below 50 copies/mL for at least the previous 6 months. At week 48 , 124 raltegravir recipients $(89.2 \%)$ and 116 ritonavir-boosted PI recipients $(86.6 \%)$ were still on virological success (difference $1.8 \%, 95 \% \mathrm{CI}$ : -5.2 to 10.6 ), reaching noninferior efficacy. The differences of efficacy results observed between the SWITCHMRK and SPIRAL switch studies strengthen the need to reliably select the eligible patients for such strategies.

The ANRS 138 EASIER trial is a prospective, randomized, open-label, noninferiority trial to compare the antiviral efficacy and safety of a switch to raltegravir with the efficacy and safety of continuing enfuvirtide. ${ }^{28,29}$ Inclusion criteria were as follows: HIV-1-infected patients with multidrugresistant virus and exhibiting plasma HIV-1 RNA levels of $<400$ copies $/ \mathrm{mL}$ for at least 3 months with an enfuvirtidebased regimen. A total of 170 patients were randomized 1:1 to maintain enfuvirtide or to switch to raltegravir. The switch to raltegravir was noninferior to the maintenance of enfuvirtide, with only one patient experiencing virological failure in each arm, leading to a difference of $0.01 \%$ between treatments (95\% CI: -6.7-6.8). At week $24,88 \%$ of patients in both arms had plasma HIV-1 RNA levels of $<50$ copies/mL. Thus, a switch from enfuvirtide to raltegravir within a virologically suppressive regimen was successful in maintaining inhibition of plasma HIV-1 replication at least over 24 weeks. A longterm follow up is needed to accurately confirm the sustained efficacy of this strategy.

\section{HIV genetic diversity and antiretroviral activity of raltegravir}

HIV is characterized by high level of genetic diversity, within HIV-1 and HIV type 2 (HIV-2). HIV-1 is subdivided into four groups: $\mathrm{M}$ (major), $\mathrm{O}$ (outlier), $\mathrm{N}$ (non-M/non-O), and $\mathrm{P}$.

Despite $40 \%$ of heterogeneity between the HIV-1 and HIV-2 integrase genes, the in vitro phenotypic susceptibility of HIV-2 clinical isolates to raltegravir was found in similar range to that of HIV- $1 .{ }^{30}$ In addition, previous studies reported a potent in vivo clinical and virological efficacy of raltegravir in HIV-2 infected patients. ${ }^{31,32}$

HIV-1 group O (HIV-O) is endemic in Western Central Africa, including Cameroon, where the prevalence of this group is estimated at about $1 \%$ of all HIV infections. HIV-O displays strong genetic divergence from HIV-1 group $\mathrm{M}$ and a high degree of intragroup diversity. A study assessing integrase polymorphism of 117 HIV-O clinical samples suggests that HIV-O presents natural mutations associated with in vitro or in vivo resistance to integrase inhibitors in
HIV-1 group M genetic context. ${ }^{33}$ However, preliminary data reported on in vivo virological response in a few number of HIV-O-infected patients receiving raltegravir-based regimen. ${ }^{34,35}$

\section{Safety and tolerability}

Overall, tolerability of raltegravir in clinical trials was excellent, and the toxicity profile of this drug is nonoverlapping with other agents, with no clear neuropsychiatric, gastrointestinal, or metabolic toxicity.

In the protocol 004, adverse events were infrequent, of mild to moderate intensity, with no obvious difference between the treatment arms. ${ }^{15,19}$

In the STARTMRK studies, significantly more patients on efavirenz than on raltegravir had clinical adverse events that were judged to be drug-related. Post-hoc analysis showed that $16 \%$ and $32 \%$ of patients on raltegravir and efavirenz, respectively, had drug-related clinical adverse events of moderate-to-severe intensity $(P<0.0001){ }^{20,22}$ Specific drug-related clinical adverse events of any severity occurring in more than $10 \%$ of participants on either raltegravir or efavirenz were dizziness, headache, and abnormal dreams. Immune reconstitution syndrome was reported as an adverse event in 17 (6\%) raltegravir recipients and 11 (4\%) efavirenz recipients. Analysis at week 8 showed that at least one central nervous system (CNS)-related adverse event had occurred in $10 \%$ of patients on raltegravir vs $18 \%$ of those on efavirenz $(P=0.0149)$. Serious adverse events occurred at a similar frequency in both treatment groups. Fewer laboratory-associated adverse events were recorded in patients on raltegravir than on efavirenz, but the difference was not significant. At week 48 , the mean changes from baseline in total cholesterol, LDLcholesterol, HDL-cholesterol, and triglyceride concentrations were smaller for raltegravir than for efavirenz recipients and the change in the total cholesterol/HDL-cholesterol ratio between treatment groups was not significant. At week 96 of the STARTMRK studies, fewer drug-related clinical adverse events $(47 \%$ vs $78 \% ; P<0.001)$ occurred in raltegravir than in efavirenz recipients. Both regimens had modest effects on serum lipids and glucose levels and on body fat composition. Consistent with the week 48 findings, mean changes from baseline in total cholesterol, LDL-cholesterol, HDL cholesterol, and triglyceride levels were smaller in raltegravir than in efavirenz recipients at week $96(P<0.001$ for each comparison). ${ }^{22}$ Changes in fat content at each time point were comparable in both treatment groups. Relatively few patients who had not developed drug-related clinical adverse events after 48 weeks of the study later developed such side 
effects. Serious adverse events and discontinuations due to adverse events were comparably infrequent in both treatment groups.

The protocol 005 and the BENCHMRK studies were not the most appropriate trials to assess the safety profile of raltegravir because all participants included in these studies previously received complex regimens and were highly pretreated. ${ }^{23-25}$ However, side effects and laboratory abnormalities were balanced between the groups in both trials, with no significant difference between the study arms. Of note, no dose-related toxicities were reported in the Phase II protocol 005 dose-ranging trial. ${ }^{23}$ In the BENCHMRK studies, a disproportionate diagnosis of several cancers was observed in the raltegravir groups (1.3\%) as compared with the placebo groups $(0.3 \%)$ at the time of the 16 -week analyses. ${ }^{24,25}$ Following these preliminary results, a global analysis including all Phase II and Phase III raltegravir studies, as well as data from the expanded access program, was performed, showing an adjusted risk of malignancy per 100 patient-years not significantly different $(2.5 \%$ in the raltegravir group vs $1.9 \%$ in the group of control subjects; Merck).

The ANRS 139 TRIO trial was an open trial without control arm, so it is difficult to identify which adverse events were related to the investigational drugs: raltegravir, etravirine, and darunavir boosted with ritonavir. ${ }^{36}$ In this study, grade 3 or 4 clinical adverse events were reported in 15 patients $(14.6 \%)$ and only one patient discontinued the regimen because of an adverse event. This combination was well tolerated, most drug-related adverse events proved to be mild or moderate in severity.

The SWITCHMRK studies, assessing the switch from lopinavir to raltegravir in virologically suppressed patients, are of interest because two of the three primary endpoints of the study carried out the safety profile: i) the mean percentage change in serum lipid concentrations from baseline to week 12 and ii) the frequency of adverse events up to 24 weeks. ${ }^{30}$ At week 12, percentage changes in lipid concentrations (total cholesterol, non-HDL cholesterol, and triglycerides) from baseline were significantly greater $(P<0.0001)$ in raltegravir recipients than in lopinavir recipients. In the combined analysis, the changes in lipid concentrations for the raltegravir group compared with the lopinavir group were $-12.6 \%$ vs $1.0 \%$ for total cholesterol, $-15.0 \%$ vs $2.6 \%$ for non-HDL cholesterol, and $-42.2 \%$ vs $6.2 \%$ for triglycerides. Changes in LDL cholesterol and HDL cholesterol were similar in the raltegravir and lopinavir groups. Clinical and laboratory adverse events occurred at similar frequencies in both treatment groups. There were no serious drug-related adverse events or deaths. Overall, virologically suppressed patients switching to raltegravir had significantly greater reductions in some lipid parameters such as total cholesterol and triglycerides. Similarly, the SPIRAL study demonstrated that switching to raltegravir was associated with significant decreases in plasma lipids (triglycerides, total, LDL, and HDL cholesterols; $P<0.0001$ for each comparison) and total-to-HDL cholesterol ratio $(P<0.05)$ relative to continuing ritonavir-boosted PI. In term of adverse events, their overall incidence was similar in the raltegravir group than in the PI group.

In the ANRS 138 EASIER trial, grade 3-4 adverse events and laboratory abnormalities were uncommon, not different between the treatment arms, and there was no difference in glucose levels between treatment arms. ${ }^{28}$

Thus, in the different studies, raltegravir had a favorable safety and tolerability profile compared with efavirenz in antiretroviral-naïve patients. In the clinical situation of a switch in virologically suppressed patients receiving a boosted PI-based regimen, an improvement of the lipid profile was observed. Overall, when analyzing the Phase II and III trials together, only a few patients on raltegravir discontinued the drug for adverse events.

\section{HIV resistance to raltegravir}

The development of resistance to raltegravir is associated with the selection of mutations in its viral target: integrase gene. Three major raltegravir resistance-associated mutations are characterized and frequently detected in vivo in case of virological failure on a raltegravir-containing regimen: Q148H/K/R, N155H, and Y143C/H. ${ }^{24,37}$ All these positions are located near the catalytic site of the enzyme. A recent crystal structure study enabled to describe the interactions between HIV-1 integrase residue Tyr 143 and the methyloxadiazole group of raltegravir, which could explain the role of the $\mathrm{Y} 143 \mathrm{C} / \mathrm{H} / \mathrm{R}$ mutations in the development of resistance to raltegravir. ${ }^{14}$ The presence of any of these key resistance mutations is sufficient to reach high level of phenotypic resistance to raltegravir (at least fold change $>15$ ). ${ }^{38}$ Furthermore, in vitro selection experiments under drug pressure showed the rapid appearance of virus with a high level of phenotypic resistance to raltegravir. ${ }^{39,40}$ Thus, raltegravir is considered as a molecule with a low genetic barrier to resistance. In most cases, the development of key raltegravir resistance-associated mutations is followed by the selection of secondary mutations specific to the resistance genetic pathway. The secondary mutations G140A/S, E92Q, and T97A 
are preferentially linked to the Q148, N155, and Y143 genetic pathways, respectively. ${ }^{40,41}$ Several studies reported that these primary resistance mutations $\mathrm{Y} 143 \mathrm{C} / \mathrm{R}, \mathrm{Q} 148 \mathrm{H} / \mathrm{K} / \mathrm{R}$, and $\mathrm{N} 155 \mathrm{H}$ represent mutually exclusive and nonoverlapping genotypic resistance pathways. ${ }^{41-43}$

In the BENCHMRK studies, a total of 105 of the 462 patients receiving raltegravir $(23 \%)$ had virological failure by week $48 .{ }^{24}$ Among these samples, integrase genotyping performed both at baseline and after virological failure showed that $68 \%$ had genotypic evidence of viral resistance to raltegravir. Virological failure was generally associated with mutations at one of the three residues: Y143, Q148, or N155, usually in combination with at least one other mutation. ${ }^{24}$

In the STARTMRK studies, in case of virological failure, genotypic resistance tests were performed only in samples with plasma HIV-1 RNA levels of $>400$ copies $/ \mathrm{mL}$. Among the eight eligible samples for this analysis in the raltegravir arm, four displayed resistant virus with key raltegravir resistance-associated mutations at positions 143 , 148, and $155{ }^{20,22}$ Between weeks 48 and 96, 12 additional patients met the protocol definition of virological failure in the raltegravir group, and 4 had plasma HIV-1 RNA level of $>400$ copies $/ \mathrm{mL}$. None of the viruses from the four evaluable raltegravir recipients had detectable resistance to any of the drugs in their regimen. ${ }^{22}$ Of note, in both BENCHMRK and STARTMRK studies, integrase genotyping was performed only in patients exhibiting HIV-1 RNA level $>400$ copies/mL for technical reasons; however, the selection of raltegravir resistance mutations has been previously described in the context of low-level viremia, between 100 and 400 copies/mL..$^{43-45}$

In the ANRS 139 TRIO trial, among the 103 patients included in the study, 14 had a virological failure, defined as a plasma HIV-1 RNA level $>50$ copies $/ \mathrm{mL}$ at week 24 or between week 24 and week 48 on two consecutive specimens for those undetectable at week $24 .{ }^{36} \mathrm{HIV}-1$ RNA level at time of virological failure was low with a median of 90 copies $/ \mathrm{mL}$. No raltegravir resistance-associated mutations were detected at time of virological failure either by bulk sequencing or by clonal analyses, performed in three subject, despite adequate plasma drug concentrations in almost all patients. ${ }^{46}$

In the ANRS 138 EASIER trial, no raltegravir resistance mutations was detected by direct sequencing in the only patient with virological failure according to the trial criteria. ${ }^{28}$ However, 39 (29\%) of the patients in the raltegravir arm of the EASIER study displayed at least one episode of low-level viremia on treatment and significant integrase resistance-associated mutations were detected in three subject (7.7\%), including $\mathrm{N} 155 \mathrm{H}$ in two subjects and P145S in one subject. ${ }^{45}$

In the SWITCHMRK studies, genotypic resistance testing was done in 14 of 16 patients with confirmed virological failure and HIV-1 RNA $>400$ copies $/ \mathrm{mL} \cdot{ }^{30}$ Of the 11 assessable patients who displayed a viral rebound on raltegravir-based therapy, virus with mutations known to confer raltegravir resistance was found in eight patients: $\mathrm{N} 155 \mathrm{H}(\mathrm{n}=6) ; \mathrm{Q} 148 \mathrm{H} / \mathrm{K} / \mathrm{R} \pm \mathrm{G} 140 \mathrm{~S}(\mathrm{n}=2) ; \mathrm{Y} 143 \mathrm{C}(\mathrm{n}=1)$. In five of these eight patients, resistance mutations were also found in the reverse transcriptase. Of note, the studies were terminated at week 24 because of lower than expected virological efficacy in the raltegravir group compared with the lopinavir group..$^{30}$

Interestingly, in the drug class of integrase inhibitors, several studies reported on the replacement of one resistant pathway by another one in patients continuing on a failing raltegravir-containing regimen. ${ }^{41,42,47}$ Most of these shifts in raltegravir-resistance profiles were characterized by the loss of variants containing $\mathrm{N} 155 \mathrm{H}$ and the emergence of variants containing Q148R/H or, in a few cases, $\mathrm{Y} 143 \mathrm{C} / \mathrm{R} \cdot{ }^{41,42,47}$ Phenotypic studies assessing viral replicative capacity and phenotypic resistance levels of raltegravir-resistant viruses showed that among single mutants, the $\mathrm{N} 155 \mathrm{H}$ had the highest selective-advantage profile. ${ }^{48}$ Among raltegravirresistant double mutants, the highest selective-advantage profile was seen with G140S + Q148H..$^{48}$ This finding likely explains why $\mathrm{N} 155 \mathrm{H}$ can be selected early in the course of raltegravir resistance evolution in vivo but is later replaced by genotypes of the Q148 pathway. The same mechanism was described for the Y143 resistance pathway. Indeed, the level of phenotypic resistance to raltegravir associated with $\mathrm{N} 155 \mathrm{H}$ is always much lower than that associated with Q148 or Y143 mutation ( $>100$ times higher). ${ }^{49}$ Moreover, the characterization of the phenotypic evolution showed that a switch from N155H to Y143C/R was linked to an increase in resistance to raltegravir. ${ }^{50}$ Taken together, these findings showed that these changes in phenotype may help to explain the shifts in integrase genotype under raltegravir treatment failure observed in different studies. . $1,42,47,49$

Little is known about the role of integrase-mutated minority variants in the development of resistance to raltegravir. Previous studies assessed the presence of such quasispecies at the baseline of raltegravir-based regimen, using different sensitive techniques (ultra-deep sequencing, allele-specific PCR assay). Thus, integrase-mutated minority 
variants were detected in some cases in minority proportions $(<1 \%)$ at baseline; however, no impact of their presence on the virological outcome was evidenced. ${ }^{51-53}$

A question remains unclear; several studies actually described the absence of selection of raltegravir resistanceassociated mutations in patients exhibiting low-level viremia on raltegravir-based therapy. ${ }^{46,54,55}$ In all Phase II and Phase III clinical trials, only the current characterized raltegravir resistance-associated mutations are reported, but we can hypothesize that the presence of other determinants, either in the integrase region or located in other parts of the pol gene, could impact on resistance.

\section{Conclusions, place of raltegravir in therapy}

The international guidelines recommend the use of raltegravir in different stages of HIV infection, corresponding to distinct profiles of clinical situations, as we described in this paragraph.

Guidelines from the European AIDS Clinical Society (EACS) include raltegravir (400 $\mathrm{mg}$ twice daily) as an alternative in the first-line regimen in antiretroviralnaïve patients (http://www.europeanaidsclinicalsociety. org/guidelinespdf). Regarding the use of raltegravir, the recently updated French 2010 recommendations (see http://www.sante-sports.gouv.fr/IMG/pdf/Prise_en_ charge_medicale_des_personnes_infectees_par_le_VIH. pdf) were similar to the EACS ones. According to the recommendations of the Department of Health and Human Services, United States guidelines (see http://aidsinfo. nih.gov/contentfiles/AdultandAdolescentGL.pdf), raltegravir (400 mg twice daily) associated with tenofovir and emtricitabine is indicated as the preferred regimen for the first-line ART.

About the use of raltegravir in a switch strategy in patients virologically suppressed, the EACS switch strategy indicates that switching from a ritonavir-boosted protease inhibitor to raltegravir in a antiretroviral-based regimen is only possible if (1) there is no history of prior virological failure with plasma virus exhibiting NRTI resistance-associated mutations and (2) NRTI backbone is fully active according to the different genotypic resistance tests available during the whole therapeutic history of the patient. A switch from enfuvirtide to raltegravir for simplification and adherence facilitation can also be considered in patients with HIV-1 RNA $<50$ copies $/ \mathrm{mL}$, following similar criteria to that described earlier for a ritonavir-boosted PI. Of note, the association of only one NRTI with raltegravir is not recommended by the EACS.

In summary, raltegravir is a potent and well-tolerated antiretroviral drug, active in vivo against a large variety of HIV strains (HIV-1 group M non-B subtypes, HIV-1 group O, HIV-2) and with many possible clinical uses:

1. in antiretroviral-naïve patients (associated with tenofovir and emtricitabine)

2. in antiretroviral-experienced patients in virological failure, associated with at least two fully active drugs

3. in antiretroviral-experienced patients in virological success (HIV-1 RNA < 50 copies/mL), associated with at least two fully active drugs and with no prior history of virological failure

In conclusion, the use of raltegravir had improved the clinical management of HIV-1 infection both in antiretroviralnaïve and in antiretroviral-experienced patients. Ongoing clinical trials currently assess new therapeutic strategies such as antiretroviral bitherapy of raltegravir combined with a PI and a new therapeutic scheme with a daily-dosing strategy. Results from ongoing trials will also help to delineate the place of raltegravir in other clinical contexts, such as the particular case of post-exposure prophylaxis, the treatment of children or adolescents infected with HIV, pregnant women, and adults co-infected with HIV and tuberculosis.

\section{Disclosure}

The authors report no conflicts of interest in this work.

\section{References}

1. Hammer SM, Saag MS, Schechter M, et al. Treatment for adult HIV infection: 2006 recommendations of the International AIDS Society-USA panel. JAMA. 2006;296(7):827-843.

2. Palella FJ Jr, Delaney KM, Moorman AC, et al. Declining morbidity and mortality among patients with advanced human immunodeficiency virus infection. HIV Outpatient Study Investigators. $N$ Engl J Med. 1998;338(13):853-860.

3. Tozzi V, Zaccarelli M, Bonfigli S, et al. Drug-class-wide resistance to antiretrovirals in HIV-infected patients failing therapy: prevalence, risk factors, and virological outcome. Antivir Ther. 2006;11(5): 553-560.

4. Costagliola D, Descamps D, Assoumou L, et al. Prevalence of HIV-1 drug resistance in treated patients: a French nationwide study. $J$ Acquir Immune Defic Syndr. 2007;46(1):12-18.

5. Hazuda DJ, Felock P, Witmer M, et al. Inhibitors of strand transfer that prevent integration and inhibit HIV-1 replication in cells. Science. 2000;287(5453):646-650.

6. Espeseth AS, Felock P, Wolfe A, et al. HIV-1 integrase inhibitors that compete with the target DNA substrate define a unique strand transfer conformation for integrase. Proc Natl Acad Sci U S A. 2000;97(21): 11244-11249.

7. Craigie R. HIV integrase, a brief overview from chemistry to therapeutics. J Biol Chem. 2001;276(26):23213-23216. 
8. LaFemina RL, Schneider CL, Robbins HL, et al. Requirement of active human immunodeficiency virus type 1 integrase enzyme for productive infection of human T-lymphoid cells. J Virol. 1992;66(12): 7414-7419.

9. Hazuda D, Blau CU, Felock P, et al. Isolation and characterization of novel human immunodeficiency virus integrase inhibitors from fungal metabolites. Antivir Chem Chemother. 1999;10(2):63-70.

10. Hazuda DJ, Anthony NJ, Gomez RP, et al. A naphthyridine carboxamide provides evidence for discordant resistance between mechanistically identical inhibitors of HIV-1 integrase. Proc Natl Acad Sci USA. 2004; 101(31):11233-11238

11. Pais GC, Zhang X, Marchand C, et al. Structure activity of 3-aryl-1,3-diketo-containing compounds as HIV-1 integrase inhibitors. J Med Chem. 2002;45(15):3184-3194.

12. Embrey MW, Wai JS, Funk TW, et al. A series of 5-(5,6)-dihydrouracil substituted 8 -hydroxy-[1,6]naphthyridine-7-carboxylic acid 4-fluorobenzylamide inhibitors of HIV-1 integrase and viral replication in cells. Bioorg Med Chem Lett. 2005;15(20):4550-4554.

13. Espeseth AS, Felock P, Wolfe A, et al. HIV-1 integrase inhibitors that compete with the target DNA substrate define a unique strand transfer conformation for integrase. Proc Natl Acad Sci U S A. 2000;97(21): 11244-11249.

14. Hare S, Gupta SS, Valkov E, Engelman A, Cherepanov P. Retroviral intasome assembly and inhibition of DNA strand transfer. Nature. 2010 464(7286):232-236.

15. Markowitz M, Nguyen BY, Gotuzzo E, et al. Rapid and durable antiretroviral effect of the HIV-1 integrase inhibitor raltegravir as part of combination therapy in treatment-naive patients with HIV-1 infection: results of a 48-week controlled study. J Acquir Immune Defic Syndr. 2007;46(2):125-133.

16. Iwamoto M, Wenning LA, Petry AS, et al. Safety, tolerability, and pharmacokinetics of raltegravir after single and multiple doses in healthy subjects. Clin Pharmacol Ther. 2008;83(2):293-299.

17. Wenning LA, Hanley WD, Brainard DM, et al. Effect of rifampin, a potent inducer of drug-metabolizing enzymes, on the pharmacokinetics of raltegravir. Antimicrob Agents Chemother. 2009;53(7): 2852-2856.

18. Iwamoto M, Wenning LA, Mistry GC, et al. Atazanavir modestly increases plasma levels of raltegravir in healthy subjects. Clin Infect Dis. 2008;47(1):137-140.

19. Markowitz M, Nguyen BY, Gotuzzo E, et al. Sustained antiretroviral effect of raltegravir after 96 weeks of combination therapy in treatmentnaive patients with HIV-1 infection. J Acquir Immune Defic Syndr. 2009;52(3):350-356

20. Lennox JL, DeJesus E, Lazzarin A, et al. Safety and efficacy of raltegravir-based versus efavirenz-based combination therapy in treatment-naive patients with HIV-1 infection: a multicentre, double-blind randomised controlled trial. Lancet. 2009;374(9692): 796-806.

21. Metzner KJ, Allers K, Rauch P, Harrer T. Rapid selection of drugresistant HIV-1 during the first months of suppressive ART in treatmentnaive patients. AIDS. 2007;21(6):703-711.

22. Lennox JL, Dejesus E, Berger DS, et al. Raltegravir versus efavirenz regimens in treatment-naive HIV-1-infected patients: 96-week efficacy, durability, subgroup, safety, and metabolic analyses. J Acquir Immune Defic Syndr. 2010;55(1):39-48.

23. Grinsztejn B, Nguyen BY, Katlama C, et al. Safety and efficacy of the HIV-1 integrase inhibitor raltegravir (MK-0518) in treatmentexperienced patients with multidrug-resistant virus: a phase II randomised controlled trial. Lancet. 2007;369(9569):1261-1269.

24. Cooper DA, Steigbigel RT, Gatell JM, et al. Subgroup and resistance analyses of raltegravir for resistant HIV-1 infection. $N$ Engl J Med. 2008;359(4):355-365.

25. Steigbigel RT, Cooper DA, Kumar PN, et al. Raltegravir with optimized background therapy for resistant HIV-1 infection. N Engl J Med. 2008; 359(4):339-354
26. Steigbigel RT, Cooper DA, Teppler H, et al. Long-term efficacy and safety of raltegravir combined with optimized background therapy in treatment-experienced patients with drug-resistant HIV infection: week 96 results of the BENCHMRK 1 and 2 Phase III trials. Clin Infect Dis. 2010;50(4):605-612.

27. Martínez E, Larrousse M, Llibre JM, et al. Substitution of raltegravir for ritonavir-boosted protease inhibitors in HIV-infected patients: the SPIRAL study. AIDS. 2010;24(11):1697-1707.

28. de Castro N, Braun J, Charreau I, et al. Switch from enfuvirtide to raltegravir in virologically suppressed multidrug-resistant HIV-1-infected patients: a randomized open-label trial. Clin Infect Dis. 2009;49(8):1259-1267.

29. Roquebert B, Damond F, Collin G, et al. HIV-2 integrase gene polymorphism and phenotypic susceptibility of HIV-2 clinical isolates to the integrase inhibitors raltegravir and elvitegravir in vitro. JAntimicrob Chemother. 2008;62(5):914-920.

30. Eron JJ, Young B, Cooper DA, et al. Switch to a raltegravir-based regimen versus continuation of a lopinavir-ritonavir-based regimen in stable HIV-infected patients with suppressed viraemia (SWITCHMRK 1 and 2): two multicentre, double-blind, randomised controlled trials. Lancet. 2010;375(9712):396-407.

31. Damond F, Lariven S, Roquebert B, et al. Virological and immunological response to HAART regimen containing integrase inhibitors in HIV-2infected patients. AIDS. 2008;22(5):665-666.

32. Garrett N, Xu L, Smit E, Ferns B, El-Gadi S, Anderson J. Raltegravir treatment response in an HIV-2 infected patient: a case report. AIDS. 2008;22(9):1091-1092.

33. Leoz M, Depatureaux A, Vessière A, et al. Integrase polymorphism and HIV-1 group O diversity. AIDS. 2008;22(10):1239-1243.

34. Briz V, Garrido C, Poveda E, et al. Raltegravir and etravirine are active against HIV type 1 group O. AIDS Res Hum Retroviruses. 2009;25(2): 225-227.

35. Charpentier C, Unal G, Depatureaux A, et al. In vivo Virological Response in HIV-1 Group O-infected Patients to Different Antiretroviral Line Regimens. International HIV and Hepatitis Virus Drug Resistance Workshop and Curative Strategies, Dubrovnik, Croatia, 2010 Jun 8-12; Abstract 111.

36. Yazdanpanah Y, Fagard C, Descamps D, et al. High rate of virologic suppression with raltegravir plus etravirine and darunavir/ritonavir among treatment-experienced patients infected with multidrug-resistant HIV: results of the ANRS 139 TRIO trial. Clin Infect Dis. 2009;49(9): 1441-1449.

37. Mouscadet JF, Delelis O, Marcelin AG, Tchertanov L. Resistance to HIV-1 integrase inhibitors: a structural perspective. Drug Resist Updat. Epub 2010 Jun 4.

38. Fransen S, Gupta S, Danovich R, et al. Loss of raltegravir susceptibility by human immunodeficiency virus type 1 is conferred via multiple nonoverlapping genetic pathways. J Virol. 2009;83(22): 11440-11446.

39. Seki T, Kobayashi M, Wakasa-Morimoto C, et al. S/GSK1349572 is a potent next generation HIV integrase inhibitor and demonstrates a superior resistance profile substantiated with 60 integrase mutant molecular clones. [Abstract 555]. 17th Conference on Retroviruses and Opportunistic Infections. San Francisco, CA, USA. $2010 \mathrm{Feb}$

40. Bar-Magen T, Sloan RD, Donahue DA, et al. Identification of novel mutations responsible for resistance to $\mathrm{MK}-2048$, a second-generation HIV-1 integrase inhibitor. J Virol. 2010;84(18):9210-9216.

41. Fransen S, Karmochkine M, Huang W, Weiss L, Petropoulos CJ, Charpentier C. Longitudinal analysis of raltegravir susceptibility and integrase replication capacity of human immunodeficiency virus type 1 during virologic failure. Antimicrob Agents Chemother. 2009;53(10): 4522-4524.

42. Malet I, Delelis O, Soulie C, et al. Quasispecies variant dynamics during emergence of resistance to raltegravir in HIV-1-infected patients. J Antimicrob Chemother. 2009;63(4):795-804. 
43. Charpentier C, Karmochkine M, Laureillard D, et al. Drug resistance profiles for the HIV integrase gene in patients failing raltegravir salvage therapy. HIV Med. 2008;9(9):765-770.

44. Ghosn J, Mazet AA, Avettand-Fenoel V, et al. Rapid selection and archiving of mutation E157Q in HIV-1 DNA during short-term low-level replication on a raltegravir-containing regimen. J Antimicrob Chemother. 2009;64(2):433-434.

45. Gallien $\mathrm{S}$, Delaugerre $\mathrm{C}, \mathrm{Hu} \mathrm{Z}$, et al. Integrase inhibitor resistance mutations in treatment-experienced HIV-1-infected patients with lowlevel viremia receiving raltegravir-containing antiretroviral therapy: an ANRS 138-EASIER trial substudy. International HIV and Hepatitis Virus Drug Resistance Workshop and Curative Strategies, Dubrovnik, Croatia, 2010 Jun 8-12; Abstract 49.

46. Charpentier C, Roquebert B, Colin C, et al. Resistance analyses in highly-experienced patients failing raltegravir, etravirine and darunavir/ ritonavir regimen (ANRS 139 TRIO trial). AIDS. Epub 2010 Aug 26.

47. Miller MD, Danovich RM, Ke Y, et al. Longitudinal analysis of resistance to the HIV-1 integrase inhibitor raltegravir: results from P005, a phase II study in treatment-experienced patients. Antivir Ther. 2008;13 Suppl 3:A8.

48. Quercia R, Dam E, Perez-Bercoff D, Clavel F. Selective-advantage profile of human immunodeficiency virus type 1 integrase mutants explains in vivo evolution of raltegravir resistance genotypes. $J$ Virol. 2009;83(19):10245-10249.

49. Hatano H, Lampiris H, Fransen S, et al. Evolution of integrase resistance during failure of integrase inhibitor-based antiretroviral therapy. J Acquir Immune Defic Syndr. 2010;54(4):389-393.
50. Reigadas S, Anies G, Masquelier B, et al. The HIV-1 integrase mutations $\mathrm{Y} 143 \mathrm{C} / \mathrm{R}$ are an alternative pathway for resistance to raltegravir and impact the enzyme functions. PLoS One. 2010;5(4):e10311.

51. Charpentier C, Laureillard D, Piketty C, et al. High frequency of integrase Q148R minority variants in HIV-infected patients naïve of integrase inhibitors. AIDS. 2010;24(6):867-873.

52. Ceccherini-Silberstein F, van Baelen K, Armenia D, et al. Secondary integrase resistance mutations found in HIV-1 minority quasispecies in integrase therapy-naive patients have little or no effect on susceptibility to integrase inhibitors. Antimicrob Agents Chemother. 2010;54(9): 3938-3948

53. Liu J, Miller M, Danovich R, et al. Detection of low-frequency mutations associated with drug resistance to raltegravir before ART [Abstract 685]. 16th Conference on Retroviruses and Opportunistic Infections. Montreal; Canada. 2009 Feb.

54. Caby F, Valin N, Marcelin AG, et al. Raltegravir as functional monotherapy leads to virological failure and drug resistance in highly treatment-experienced HIV-infected patients. Scand J Infect Dis. 2010; 42(6/7):527-532.

55. Allavena C, Mounoury O, Rodallec A, et al. Raltegravir in HIV-1 ARVexperienced patients: high efficacy and absence of emergence of resistance mutations in low-grade virologic failures. [Abstract PE7.9/15]. 12th European AIDS Conference/EACS. Koln, Germany. Nov 2009
Infection and Drug Resistance

\section{Publish your work in this journal}

Infection and Drug Resistance is an international, peer-reviewed openaccess journal that focuses on the optimal treatment of infection (bacterial, fungal and viral) and the development and institution of preventive strategies to minimize the development and spread of resistance. The journal is specifically concerned with the epidemiology of antibiotic

\section{Dovepress}

resistance and the mechanisms of resistance development and diffusion in both hospitals and the community. The manuscript management system is completely online and includes a very quick and fair peerreview system, which is all easy to use. Visit http://www.dovepress.com/ testimonials.php to read real quotes from published authors. 